\title{
ON MINIMAL $n$-UNIVERSAL GRAPHS
}

\author{
by J. W. MOON
}

(Received 31 March, 1964)

A graph $G_{n}$ consists of $n$ distinct vertices $x_{1}, x_{2}, \ldots, x_{n}$ some pairs of which are joined by an edge. We stipulate that at most one edge joins any two vertices and that no edge joins a vertex to itself. If $x_{i}$ and $x_{j}$ are joined by an edge, we denote this by writing $x_{i} \circ x_{j}$.

Consider a second graph $H_{N}$, where $n \leqq N$. Following Rado [1], we say that a one-to-one mapping $f$ of the vertices of $G_{n}$ into the vertices of $H_{N}$ defines an embedding if $x_{i} \circ x_{j}$ implies $f\left(x_{i}\right) \circ f\left(x_{j}\right)$, and conversely, for all $i, j=1,2, \ldots, n$. If there exists an embedding of $G_{n}$ into $H_{N}$, we denote this by writing $G_{n} \prec H_{N}$. The particular graph $H_{N}$ is said to be $n$-universal if $G_{n} \prec H_{N}$ for every graph $G_{n}$ with $n$ vertices.

For each positive integer $n$, let $\lambda(n)$ denote the least integer $N$ for which there exists an $n$-universal graph $H_{N}$. (It is clear that $\lambda(n)$ is finite, since the graph consisting of disjoint copies of all the graphs with $n$ vertices is $n$-universal.) The object in this note is to establish the following inequalities:

$$
2^{\frac{1}{(n-1)}} \leqq \lambda(n) \leqq \begin{cases}n \cdot 2^{\frac{1}{(n-1)}} & \text { if } n \text { is odd } \\ \frac{3}{2 \sqrt{2}} n \cdot 2^{\frac{1}{(n-1)}} & \text { if } n \text { is even. }\end{cases}
$$

The first inequality is obtained by the following simple argument. There are at least $2^{\left(\begin{array}{l}n \\ 2\end{array}\right)} / n$ ! different graphs $G_{n}$, since the labellings assigned to the vertices are immaterial to the problem. Hence, if $H_{N}$ is $n$-universal,

$$
2^{\left(\begin{array}{l}
n \\
2
\end{array}\right) / n ! \leqq}\left(\begin{array}{l}
N \\
n
\end{array}\right) \leqq N^{n} / n !
$$

since different graphs $G_{n}$ must be mapped onto different subgraphs with $n$ vertices of $H_{N}$. The lower bound for $\lambda(n)$ now follows immediately. Slight improvements may be obtained by using better estimates for the number of different graphs $G_{n}$.

To obtain an upper bound for $\lambda(n)$ we proceed as follows. Let $T_{n}$ be any oriented complete graph with $n$ vertices $y_{1}, y_{2}, \ldots, y_{n}$. Write $y_{i} \rightarrow y_{j}$ if the edge joining $y_{i}$ and $y_{j}$ is oriented from $y_{i}$ to $y_{j}(i \neq j)$. Let $Y_{i}=\left\{y_{j}: y_{j} \rightarrow y_{i}\right\}$. Construct a graph $H$ whose vertices $z_{i, A}$ are in one-to-one correspondence with the ordered pairs $(i, A)$, where $A \subset Y_{i}$. If $A \subset Y_{i}$, $B \subset Y_{j}$ and $y_{i} \rightarrow y_{j}$, then let $z_{i, A} \circ z_{j, B}$ in $H$ if and only if $y_{i} \in B$.

We now show that $H$ is $n$-universal. If $G_{n}$ has vertices $x_{1}, x_{2}, \ldots, x_{n}$, we may set $f\left(x_{i}\right)=z_{i, A(i)}$, where $A(i)=\left\{y_{j}: y_{j} \rightarrow y_{i}\right.$ and $\left.x_{j} \circ x_{i}\right\}$. Then $f$ is an embedding of $G_{n}$ into $H$, since, if $y_{i} \rightarrow y_{j}$, we have

$$
f\left(x_{i}\right) \circ f\left(x_{j}\right) \Leftrightarrow z_{i, A(i)} \circ z_{j, A(j)} \Leftrightarrow y_{i} \in A(j) \Leftrightarrow x_{i} \circ x_{j} .
$$


Therefore

$$
\lambda(n) \leqq(\text { number of vertices of } H)=2^{\left|Y_{1}\right|}+2^{\left|Y_{2}\right|}+\ldots+2^{\left|Y_{n}\right|} .
$$

To minimise this sum, let $T_{n}$ be the oriented complete graph in which $y_{i} \rightarrow y_{j}$ if and only if $0<j-i \leqq\left[\frac{1}{2} n\right]$, where the subtraction is modulo $n$ or $n+1$ according as $n$ is odd or even. For this choice of $T_{n}$ it is not difficult to see that

$$
\begin{gathered}
\left|Y_{1}\right|=\ldots=\left|Y_{n}\right|=\frac{1}{2}(n-1), \quad \text { if } n \text { is odd, } \\
\left|Y_{1}\right|=\ldots=\left|Y_{1 n}\right|=\frac{1}{2} n, \quad\left|Y_{\frac{1}{2} n+1}\right|=\ldots=\left|Y_{n}\right|, \quad \text { if } n \text { is even. }
\end{gathered}
$$

Hence

$$
\begin{gathered}
\lambda(n) \leqq n \cdot 2^{\ddagger(n-1)}, \quad \text { if } n \text { is odd, } \\
\lambda(n) \leqq \frac{1}{2} n \cdot 2^{\ddagger n}+\frac{1}{2} n \cdot 2^{\frac{\ddagger}{(n-2)}}=\frac{3}{2 \sqrt{ } 2} n \cdot 2^{\frac{1}{(2 n-1)}}, \quad \text { if } n \text { is even. }
\end{gathered}
$$

This completes the proof of the above inequalities.

I am indebted to the referee for suggestions leading to a substantial improvement in the upper bound for $\lambda(n)$.

\section{REFERENCE}

1. R. Rado, Universal graphs, Acta Arith. (to appear).

\section{UNIVERSITY COLLEGE}

LONDON

(Now at University of Alberta, Edmonton, Canada) 